\title{
Entretien avec Najeh Jegham
}

Interview with Najeh Jegham

\section{Najeh Jegham}

\section{(2) OpenEdition}

\section{Journals}

Édition électronique

URL : http://journals.openedition.org/recherchestravaux/1841

DOI : 10.4000/recherchestravaux.1841

ISSN : 1969-6434

Éditeur

UGA Éditions/Université Grenoble Alpes

Édition imprimée

ISBN : 978-2-37747-165-2

ISSN : 0151-1874

\section{Référence électronique}

Najeh Jegham, "Entretien avec Najeh Jegham », Recherches \& Travaux [En ligne], 95 | 2019, mis en ligne le 05 décembre 2019, consulté le 08 septembre 2020. URL : http://journals.openedition.org/ recherchestravaux/1841; DOI : https://doi.org/10.4000/recherchestravaux.1841

Ce document a été généré automatiquement le 8 septembre 2020.

(c) Recherches \& Travaux 


\title{
Entretien avec Najeh Jegham
}

Interview with Najeh Jegham

\author{
Najeh Jegham
}

\section{NOTE DE L'ÉDITEUR}

Najeh Jegham s'est entretenu, le 10 novembre 2017 à Grenoble, avec Atef Boussoualim, qui a enregistré puis retranscrit cet entretien qui accompagnait le colloque «L'écrivain-traducteur : ethos et style d'un co-auteur ». La version est ici enrichie des propos de l'auteur recueillis par Aline Marchand lors de la table-ronde animée par Pascale Roux, la veille, au Café des Arts, dont une captation est disponible en ligne : <https://archive.org/details/ElAmraouiGharrafijeghamCafDesArtsV2>.

Question: Votre parcours est pour le moins atypique, tout comme vos travaux: poésie, calligraphie, "callilecture », "callimuse ${ }^{1}$... les formes fusent et s'entremêlent, gardant le même préfixe «calli ». Pourquoi avoir choisi ces formes?

Najeh Jegham : Ce qui s'appelle callimuse, callilecture, est fondé sur la calligraphie, il s'agit d'un travail pour accompagner de la poésie, un travail de composition à partir de la poésie et dans la rencontre entre poésie, calligraphie et musique aussi. Mais surtout musique-calligraphie. Je considère que la poésie a une dimension visible, le poème est un texte visible, bien sûr audible, mais il a aussi cette dimension-là, ne serait-ce que parce que ça se dispose verticalement.

Q. : Le poème est-il fini une fois écrit, faut-il forcément le lire ou l'interpréter? Je me rappelle d'une phrase de Ferré où il disait : « toute poésie destinée à n'être que lue et enfermée dans sa typographie n'est pas finie; elle ne prend son sexe qu'avec la corde vocale tout comme le violon prend le sien avec l'archet qui le touche ». Est-ce qu'un texte écrit est fini?

Najeh Jegham : S'il est fini il est mort, donc il n'est jamais fini. Bon, c'est peut-être dit un peu trop vite, trop brutalement comme ça. Un poème n'a pas une seule existence, il n'a pas une seule vie. C'est aussi la fonction de l'art, de prolonger la vie et de nous prolonger, que l'on soit créateurs ou récepteurs, lecteurs, auditeurs, etc. C'est une forme peut-être, une manifestation qui est finie, mais il y a toujours la possibilité d'y 
revenir, de relire. Peut-être que l'auteur qui a produit une forme ne va pas la changer, sauf s'il passe par la traduction, il peut donner une autre manifestation à travers la traduction, une autre version qui est à la fois le même poème et autre chose, donc il est infini tout simplement et c'est le sens même de la vie.

Q. : Vous avez dit pendant le colloque que toute langue est étrangère, l'invention permanente des langues leur permet de durer dans le temps, croyez-vous qu'une langue qui ne change pas est vouée à disparaître?

Najeh Jegham : Est-ce que ça existe, une langue qui ne change pas? Sauf si elle est "morte ", comme on l'appelle. Toute langue qui existe, qui est pratiquée, est vivante ; elle change, elle vit, elle grandit, elle emprunte, elle s'accouple à d'autres, etc. comme tout vivant. Mais quand je dis que toute langue est étrangère, tout simplement parce que toute langue passe par un apprentissage, on n'a jamais fini d'apprendre une langue, la maîtrise parfaite n'existe pas, on vise peut-être la perfection mais on ne l'atteint pas et heureusement d'ailleurs [rire]. Donc même une langue maternelle réclame un apprentissage, c'est quelque chose d'étranger, même si on a une relation particulière avec cette première langue. Parce qu'elle est première, parce qu'on a baigné dedans, on l'a fréquentée plus longtemps, c'est la langue de la mère, c'est la langue de l'enfance, mais même celle-ci exige un apprentissage.

Q. : En tant qu'auteur et grand lecteur, aussi bien en arabe qu'en français, entretenez-vous la même relation à ces deux langues?

Najeh Jegham : Chronologiquement l'arabe est la première langue, c'est indiscutable, ensuite vient le français, même si en ce qui me concerne je préfère avoir une relation égalitaire avec les deux langues. Les deux langues sont miennes, parce que je considère que chaque langue est étrangère, chaque langue réclame un apprentissage. On ne naît pas avec la maîtrise linguistique, on a besoin d'un apprentissage qui se fait différemment bien sûr en ce qui concerne une langue première ou une langue seconde, mais c'est toujours un territoire étranger en quelque sorte qui nécessite d'être conquis. Lors de la table ronde, j'ai peu lu en arabe, j'écris plus en français et généralement je n'aime pas traduire, je n'aime pas me traduire. Ce qui m'intéresse beaucoup c'est la relation, le va-et-vient, le fait d'être dans une langue me pousse à convoquer d'autres langues, pour servir la première, quelle que soit la première et vice-versa. Quand on est dans la pluralité des langues, dans le bilinguisme, très souvent on observe le phénomène de traduction qui est convoqué facilement, rapidement, ne serait-ce que par jeu. Le jeu fait partie de l'humain et ça fait partie de l'enfance également, et le passage d'une langue à l'autre relève d'abord de ce jeu. D'abord par la traduction, et si on est bilingue, on observe souvent la facilité du jeu de mots aussi, parce qu'on a un rapport particulier à la langue.

Q. : Ces deux langues représentent deux univers culturels distincts et on pourrait penser que jamais elles ne pourront se rencontrer jusqu'à ce qu'on lise vos œuvres, comment arrivez-vous à les combiner et les assembler?

Najeh Jegham : Il faut peut-être relativiser cette distance entre l'arabe et le français, l'histoire est là pour les rapprocher, même si les contacts ne se sont pas, hélas! déroulés pacifiquement. Beaucoup de violences dans l'histoire, mais quand on observe aujourd'hui la pratique de l'arabe et du français, on dit facilement au Maghreb «merci » et on parle sa langue en disant «merci ». C'est la même chose quand on parle de son «bled» en français, le mot «bled» est un mot français aujourd'hui, donc ça relativise quand même la distance entre les deux langues. Même 
s'il y a des univers qui sont complétement distincts, il y a des contacts qui sont effectifs, prégnants et qui montrent que ça bouge, que ça circule. Peut-être qu'on souffre davantage de représentations qui séparent, surtout à notre époque historique, la distance s'agrandit et des discours de fermeture sont de plus en plus présents. Les murs poussent un peu partout et du coup, ça donne l'impression d'une distance grande. Mais effectivement, concrètement, on observe des contacts qui sont toujours là et qui vont durer parce que l'histoire le veut et parce que l'humain également le veut, il est difficile de séparer. Dans le vécu de la France d'aujourd'hui par exemple, l'arabe c'est la deuxième langue parlée en France, c'est ça! On ne le dit pas malheureusement, c'est une langue de France, c'est la deuxième langue pratiquée, il suffit de circuler dans les marchés pour entendre l'arabe, beaucoup font leurs courses en arabe.

Q. : Est-ce que ces deux langues sont mêlées justement? Parvenez-vous à les démêler si c'est le cas?

Najeh Jegham : Je préfère les mêler, non pas les démêler, pourquoi les démêler ? Les deux langues sont miennes. Je les revendique et revendique leur mêlée parce qu'elle est jouissive. C'est productif, c'est enrichissant et c'est agréable aussi. Tant mieux si elles sont mêlées, il ne faut surtout pas les démêler [rire]. Dans le deuxième recueil, Épars, des poèmes comprennent des mots arabes, parfois traduits mais pas toujours, et dans leur graphie arabe, qui arrivent dans un texte en français ${ }^{2}$. Ce n'est peut-être pas gentil pour le lecteur, mais c'est son affaire, peut-être que le lecteur idéal est le lecteur bilingue! [rire] Mais, en même temps, je considère qu'il faut faire avec l'incompréhension, qui fait partie de la vie, de la littérature, de la lecture. Peut-être que l'effort à faire est de pacifier ces choses-là et de ne pas arrêter une lecture parce qu'on n'a pas compris tel paragraphe, telle page ou tel chapitre. Il faut lire et la compréhension n'est pas un départ mais l'aboutissement d'une démarche, si elle est là au départ, je crois que ce n'est plus la peine de lire.

Q.: Lorsque vous écrivez en français, même vos lieux sont français, vous évoquez Carcassonne et Perpignan par exemple, ces lieux vous renvoient étrangement à des mots en arabe comme Meshmesh et Warwar dans Épars, l'inverse n'est pas vrai par contre; lorsque vous écrivez en arabe, aucun mot en français ne vient interférer dans votre écriture, comment l'expliquez-vous?

Najeh Jegham : Ça s'explique tout simplement par les circonstances de l'écriture, ce qui pousse à écrire. Les textes auxquels tu fais référence sont des textes qui ont été écrits dans un train, qui passait par Carcassonne, j'allais à Perpignan. Tout ce qui est dit dans le poème est authentique et ça montre aussi cette relation que j'aime bien avoir avec la langue. L'intérêt pour la dimension sonore. Carcassonne en tant que mot, en tant que masse sonore, ça a son importance, son pouvoir d'imitation et du coup je me suis déplacé pour voir la même chose en arabe, donc ces mots naissent d'eux-mêmes comme je l'ai écrit. Voilà, c'est cette relation un peu ludique à la langue qui m'intéresse et qui nourrit mon écriture. Si ça se manifeste davantage en français c'est parce que j'étais dans le train en France allant à Perpignan et passant par Carcassonne, voilà ! ça aurait pu arriver entre Tunis et Gafsa et ça aurait été le même phénomène mais dans l'autre sens.

Q.: Quelle posture prenez-vous lorsque vous traduisez? Restez-vous fidèle au texte d'origine en traduisant littéralement ou bien la liberté de l'écrivain est-elle souveraine?

Najeh Jegham: Je suis d'abord un lecteur et j'ai commencé à traduire en lisant, d'ailleurs j'en suis venu à la calligraphie grâce à la lecture, grâce à la poésie et 
surtout, pour être précis, grâce au poète Mahmoud Darwich, un poète palestinien. Je le lisais : il y avait des vers, des passages que je trouvais sublimes et je me disais que j'aurais aimé les écrire. Comme c'était déjà écrit, je me suis mis à calligraphier, une façon de m'approprier le texte. Dans la traduction aussi, le même fonctionnement s'est opéré, c'est-à-dire que je peux avoir besoin de traduire simplement pour prolonger d'abord la relation avec le texte qui me plaît et aussi pour le découvrir, parce que la traduction a ce pouvoir révélateur d'une signification et d'une vie du texte, c'est toujours enrichissant. J'ai commencé à traduire d'abord dans ce cadre-là, dans le cadre de la lecture tout simplement.

\section{Q. : Ce sont des traductions fidèles au texte?}

Najeh Jegham: Pour la fidélité, je préfère parler de fidélité à moi-même, à mes principes. Le principe de respect est indiscutable, pour moi, dans les relations quelles qu'elles soient; les relations humaines mais également les relations avec un texte. Je considère un texte aussi comme un être vivant, en quelque sorte, il a une histoire, il a une naissance, etc., je le respecte de ce point de vue-là. Donc la fidélité, c'est d'abord cette fidélité-là. Tout à l'heure, dans le colloque, j'ai parlé de cette notion de fidélité à l'intention artistique, que j'ai empruntée à un autre critique. Donc il faut définir la fidélité, ce n'est pas une fidélité aux mots mais une fidélité à un esprit, encore faut-il le saisir et être sensible à son établissement. C'est pour ça que je préfère parler de fidélité à moi-même. Quand je m'engage dans une activité, je m'y engage véritablement, pour servir le texte et servir ma relation au texte, le texte n'existe pas hors relation de lecture. Il n'y a pas d'écriture sans lecture, donc il s'agit d'être fidèle à cela.

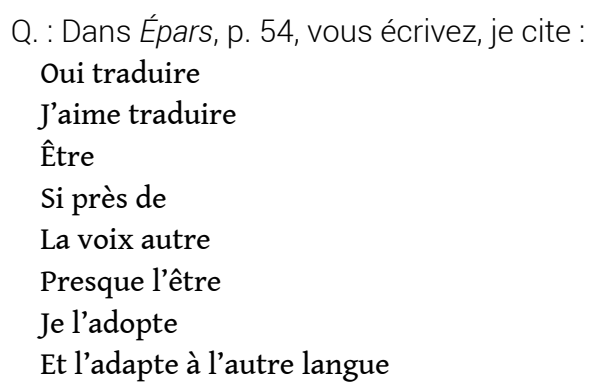

L'acte de traduire vous éloigne-t-il de votre propre personne ou bien l'autre c'est aussi vous? Ce Je de la première personne est-il multiple: le vôtre ainsi que celui de l'auteur traduit?

Najeh Jegham : Un traducteur est auteur de sa traduction, il s'approprie le texte, il lui donne une autre naissance dans l'autre langue, donc il y a une communication entre ces deux Je. Bien sûr, personne ne peut remplacer l'auteur initial, ne peut l'écarter ou prendre sa place. Sauf que dans la traduction, il y a une nouvelle écriture à partir d'un premier texte et le traducteur est responsable, maitre de sa traduction, donc il y a cette communication, là oui !

Q. : Avant l'islam, la langue arabe, à travers la poésie en particulier, a longtemps loué l'amour, le sexe, le vin et beaucoup de sujets qui peuvent être considérés comme tabous aujourd'hui. L'islam a en quelque sorte sacralisé cette langue. Pendant le colloque, on a parlé du mot « whisky » qu'on ne voulait pas traduire par exemple. Est-ce que l'arabe doit être considéré comme une langue de censure aujourd'hui ? Existe-t-il des sujets que vous ne pouvez pas aborder en arabe et que vous traitez facilement en français ?

Najeh Jegham : Une langue ne censure pas, c'est un pouvoir qui censure. La langue est d'abord un outil pour dire et on peut tout dire dans la langue. C'est une question de 
pouvoir, censurer c'est se donner le droit de dire : voilà ce qui est autorisé, voilà ce qui ne l'est pas et sur tous les plans, linguistique, comportemental, etc. La langue est innocente, il se trouve que la langue arabe est aussi la langue du Coran, c'est-à-dire une langue en rapport direct avec une notion sacrée et le sacré c'est l'absolu. Cela passe par une dimension d'intouchable, de tabou selon la logique du licite et de l'illicite. Donc ça, c'est la logique religieuse, qui est respectable en soi et il n'y a aucun problème, de mon point de vue, si quelqu'un adhère à cela. C'est la relation d'une personne avec un certain sacré, c'est quelque chose de personnel, la langue c'est autre chose.

Q. : Vous arrivez à les dissocier justement, la langue et la religion ? En d'autres termes, depuis l'arrivée de l'islam, est-ce que l'arabe peut tout se permettre ou y a-t-il une réelle censure?

Najeh Jegham : La langue n'a pas à permettre ou ne pas permettre, c'est un pouvoir qui permet ou ne permet pas. La langue est un champ de possibles. Le possible est variable bien sûr, selon les langues mais la langue arabe, plutôt que de parler de sa relation étroite avec l'islam, il faut parler d'abord de son statut de langue sémitique, de langue qui a précédé l'islam. Ce n'est pas l'islam qui a donné la langue arabe mais l'inverse. C'est l'islam qui est né en arabe, c'est important de situer chronologiquement les choses. Il y a eu d'abord la langue, la culture arabes et les arabes, ensuite il y a eu l'islam. Il faut d'abord parler de cette langue qui, à partir du moment où c'est une langue à racines, je crois qu'elle situe son locuteur dans une perspective de création: le fonctionnement à partir d'une racine et à partir de ce qu'on appelle un "schème» - je n'aime pas trop ce mot, je préfère "forme »permet de créer toutes sortes de mots. Il y a des mots qui existent et il y a des mots possibles qui ne sont pas dans l'usage mais qui peuvent exister. Je préfère parler d'abord de cette caractéristique de la langue ; comme champ de création, un champ ouvert. La religion ne s'inscrit pas dans le champ ouvert, elle s'inscrit nécessairement dans un champ fermé à partir de cette pensée de l'absolu, du sacré et de l'interdit, etc. Je ne suis pas du tout dans cette logique, je respecte celui qui est dedans, c'est son affaire, ce n'est pas la mienne. On constate que même pendant la période islamique, la poésie notamment a chanté le sexe, a chanté le vin, a chanté la vie. C'est une dimension importante qu'il ne faut pas oublier. Malheureusement, mais bon, c'est normal d'un certain point de vue, cette époque est quand même violente et l'arabe est associé à cette violence évidemment. Tout à l'heure, je parlais de «bled » mot arabe devenu français, maintenant tous les Français connaissent «Allahou Akbar » parce que celui qui tue commence par dire "Allahou Akbar", à chaque fois où on l'entend c'est qu'il y a un meurtre. Il m'est arrivé à plusieurs reprises, lors de rencontres, de dire aux Français : est-ce que vous savez comment on dit «beauté » en arabe? comment on dit «liberté » en arabe ? Ça se dit aussi en arabe mais vous ne savez dire qu' «Allahou Akbar », vous ne savez dire que «jihad ». C'est quand même regrettable, moi je le regrette. Il y a les mots « beauté », il y a les mots « vie », il y a les mots «fraternité », "égalité », et c'est vraiment regrettable de ne pas les diffuser aussi dans notre vécu collectif aujourd'hui. Encore une fois, la langue est innocente.

Q. : Pour ceux qui ne maîtrisent pas l'arabe, pouvez-vous nous dire si les deux œuvres Distances d'aimer et Masafat hubb sont la même œuvre traduite ou bien deux œuvres distinctes?

Najeh Jegham : J'aimerais dire d'abord à ceux qui ne maitrisent pas l'arabe qu'il est temps qu'ils s'y mettent [rire]. Pour revenir à cette œuvre, Distances d'aimer, c'est 
parce que justement dans ce recueil, il y a une distinction entre l'amour et aimer, mais bon, c'est un autre débat. Je présente toujours ce recueil comme un recueil en deux parties, qui a deux commencements et qui n'a pas de fin. Si on prend le livre, on a une couverture à droite en arabe. De l'autre côté, il y a une couverture en français et heureusement que la langue arabe va de droite à gauche et la langue française de gauche à droite. On a une partie complètement en arabe, de l'autre côté une autre partie complètement en français. Elles ont le même nombre de pages et les deux langues se rencontrent dans le geste esthétique de la calligraphie, qui est elle-même bilingue ou unilingue. Et les textes se parlent, dialoguent, se répondent : il y a des questions dans une partie, dans une langue, et des éléments de réponse dans l'autre. Il s'agit donc d'un dialogue entre les langues mais pas de coïncidences, parce que la coïncidence parfaite est impossible. Dans ce que j'ai lu lors de la table-ronde, j'ai tenté une traduction, parce que c'était plus agréable peut-être et pour que chacun en ait un plus grand accès. Mais je considère qu'écouter une poésie en langue étrangère, sans du tout axer sur le sens des mots, cela vaut toujours le coup, parce que le sens n'est pas seulement dans les mots, mais dans une musique, dans des sonorités, dans divers éléments qui forment aussi la poésie. Donc je préfère généralement relativiser ce qu'on peut appeler les «effets de la langue » ou la différence linguistique. Il y a, autant que possible, la même démarche dans les deux langues, je considère donc que ce recueil bilingue est un livre et non pas deux. C'est un mouvement qui se fait d'une langue à l'autre et la rencontre véritable se fait dans le geste artistique. Symboliquement, je tiens à cela, je trouve, et c'est valable pour notre actualité historique, que notre rencontre ne peut se faire véritablement que dans l'art, que dans la culture. Je constate que ce qui est frappé à notre époque, c'est justement la culture. Je me dis parfois, quand ça va mal : pourquoi c'est l'Irak et la Syrie qu'on est en train de détruire ? Il se trouve que l'histoire est née en Irak, l'écriture est née en Irak, l'histoire commence par l'invention de l'écriture, Sumer c'est l'actuel Irak. La Syrie, la Phénicie comme on l'appelait, c'est la naissance de l'alphabet. C'est quand même étonnant que ces destructions se situent là, ce n'est pas la théorie du complot mais c'est un constat qui a sa pertinence. En tout cas, je reviens à ce qui m'intéresse, c'est cette rencontre qui doit se faire et qui ne peut se faire, à mon sens, que dans la culture et l'art. Dans cet ouvrage, cette distance d'aimer c'est la distance qui sépare aussi ces deux commencements du livre et la rencontre se fait dans la calligraphie qui est à la fois une manifestation de la graphie, de l'écriture donc de la langue, ou d'un aspect de la langue et en même temps, c'est un geste artistique. Il n'est pas nécessaire de connaître l'arabe pour apprécier une calligraphie arabe, on apprécie facilement une calligraphie chinoise. Je ne lis pas le chinois mais je suis très sensible à cela. Il y a autre chose, il y a l'aspect formel, il y a les compositions, etc., voilà ce que je veux mettre en avant.

\section{Q. : Pour conclure, pouvez-vous nous dire quels sont vos projets?}

Najeh Jegham : Mes projets? Je peux parler d'un projet, j'en ai parlé hier après la lecture qui a eu lieu, c'est de réaliser véritablement une œuvre bilingue. C'est un vieux rêve et c'est un projet dans lequel je me suis engagé il y a des années. Il y a donc les deux recueils que j'ai publiés qui situent des étapes, des tentatives, je dirais, de réaliser cette œuvre bilingue. Mais mon projet c'est surtout d'écrire un roman bilingue, un roman qui soit trois romans à la fois, un roman dans lequel le lecteur 
unilingue francophone trouve vraiment une unité en français, le lecteur arabophone pareil, qu'il puisse lire vraiment une histoire dans sa langue.

\section{Q. : La même histoire ou une autre?}

Najeh Jegham: Deux histoires qui communiquent ou une histoire traitée selon différents aspects, différents points de vue et que l'ensemble constitue une unité. C'est ça le défi dans lequel je me suis engagé depuis longtemps. Ça réclame du temps et malheureusement il y a beaucoup de contraintes qui m'empêchent de me consacrer à cela. En tout cas, ce sont toujours ces questions qui m'intéressent.

\section{NOTES}

1. Néologismes forgés par l'auteur pour définir ses créations littéraires et artistiques

2. N. Jegham, Épars, Saint-Denis, Édilivre, 2016 ; voir notamment p. 71 et 86.

\section{RÉSUMÉS}

Dans cet entretien de novembre 2017, poursuivi lors d'une table-ronde au Café des Arts à Grenoble, Najeh Jegham évoque le geste et le souffle que ses poèmes partagent avec l'art de la calligraphie ou avec la musique - en témoignent ses « callilectures » et « callimuses ». Ainsi, il ne présente pas son recueil de poèmes et de calligraphies bilingues, Distances d'aimer (L'aile éditions, 2004), comme un volume bilingue résultant d'une auto-traduction mais comme constitué de deux textes (arabe et français), autonomes, qui se rencontrent dans le geste calligraphique. De même, dans son recueil poétique Épars (L'aile éditions, 2016), des poèmes écrits en français incluent des mots arabes dans leur graphie, permettant au lecteur non arabophone d'expérimenter cette incompréhension première face à la langue. Concevant la traduction et la calligraphie comme un même geste de relecture, Najeh Jegham fait l'éloge d'un rapport ludique à la langue, loin de toute censure politique ou religieuse.

In this interview in November 2017, continued during a round table discussion at the Cafe des Arts in Grenoble, Najeh Jegham evokes the gesture and the breath that his poems share with the art of calligraphy or with music - as evidenced by his "callilectures" and "callimuses". Thus, he does not present his collection of bilingual poems and calligraphies, Distances d'aimer (L'aile éditions, 2004), as a bilingual volume resulting from self-translation but as two autonomous texts (Arabic and French), which meet in the calligraphic gesture. Similarly, in his poetic book Épars (L'aile éditions, 2016), poems written in French include Arabic words in their writing, allowing non-Arabic-speaking readers to experience this first misunderstanding of the language. Conceiving translation and calligraphy as the same gesture of rereading, Najeh Jegham praises a playful relationship with language, far from any political or religious censorship. 